\title{
BMJ Open Queensland Family Cohort: a study protocol
}

To cite: Borg D, Rae K, Fiveash C, et al. Queensland Family Cohort: a study protocol. BMJ Open 2021;11:e044463. doi:10.1136/ bmjopen-2020-044463

- Prepublication history and additional supplemental material for this paper are available online. To view these files, please visit the journal online (http://dx.doi.org/10.1136/ bmjopen-2020-044463).

$\mathrm{DB}$ and $\mathrm{KR}$ contributed equally.

Received 16 September 2020 Accepted 23 April 2021

Check for updates

(C) Author(s) (or their employer(s)) 2021. Re-use permitted under CC BY-NC. No commercial re-use. See rights and permissions. Published by BMJ.

For numbered affiliations see end of article.

Correspondence to

Dr Vicki Clifton;

vicki.clifton@mater.uq.edu.au

Danielle Borg (D) ,1,2 Kym Rae,, ${ }^{1,2}$ Corrine Fiveash, ${ }^{3}$ Johanna Schagen, ${ }^{1,2}$ Janelle James-McAlpine, ${ }^{1,2}$ Frances Friedlander, ${ }^{4}$ Claire Thurston, ${ }^{1,2}$ Maria Oliveri, ${ }^{1,2}$ Theresa Harmey, ${ }^{1,2}$ Erika Cavanagh, ${ }^{5,6,7}$ Christopher Edwards, ${ }^{5,7}$ Davide Fontanarosa, ${ }^{5,7}$ Tony Perkins, ${ }^{8}$ Greig de Zubicaray, ${ }^{5,7}$ Karen Moritz, ${ }^{2,9}$ Sailesh Kumar (D) , ${ }^{1,2}$ Vicki Clifton, ${ }^{1,2}$ Queensland Family Cohort Research Collaborative

\section{ABSTRACT}

Introduction The perinatal-postnatal family environment is associated with childhood outcomes including impacts on physical and mental health and educational attainment. Family longitudinal cohort studies collect in-depth data that can capture the influence of an era on family lifestyle, mental health, chronic disease, education and financial stability to enable identification of gaps in society and provide the evidence for changes in government in policy and practice.

Methods and analysis The Queensland Family Cohort (QFC) is a prospective, observational, longitudinal study that will recruit 12500 pregnant families across the state of Queensland (QLD), Australia and intends to follow-up families and children for three decades. To identify the immediate and future health requirements of the QLD population; pregnant participants and their partners will be enrolled by 24 weeks of gestation and followed up at 24, 28 and 36 weeks of gestation, during delivery, on-ward, 6 weeks postpartum and then every 12 months where questionnaires, biological samples and physical measures will be collected from parents and children. To examine the impact of environmental exposures on families, data related to environmental pollution, household pollution and employment exposures will be linked to pregnancy and health outcomes. Where feasible, data linkage of state and federal government databases will be used to follow the participants long term. Biological samples will be stored long term for future discoveries of biomarkers of health and disease.

Ethics and dissemination Ethical approval has been obtained from the Mater Research Ethics (HREC/16/ MHS/113). Findings will be reported to (1) QFC participating families; (2) funding bodies, institutes and hospitals supporting the QFC; (3) federal, state and local governments to inform policy; (4) presented at local, national and international conferences and (5) disseminated by peer-review publications.

\section{BACKGROUND AND RATIONALE}

The Developmental Origins of Health and Disease (DOHaD) proposes that the intrauterine environment, when impacted by poor maternal nutrition, severe stress or illness, has a detrimental impact on the developing
Strengths and limitations of this study

- In-depth data and biological sample collection of physical, social and mental well-being of mother, father/partner and child at multiple time points.

- Survey instruments based on validated tools and routinely collected clinical data.

- Recognising the equal importance of the family relationships (including same sex relationships) on the child so that mothers, fathers or partners are all involved.

- Cultural consultation for individuals who identify as First Nations People is ongoing.

- Cost to maintain cohort, infrastructure, storage of data and biobank is high, with lifelong surveillance of families dependent on philanthropic, state and/or federal support.

child, both in the short and long terms. These prenatal effects can increase the risk of cardiovascular disease, hypertension, obesity, type 2 diabetes, metabolic syndrome and kidney disease ${ }^{1-3}$ However, as the scientific community learns more about how health develops in adulthood, we are also recognising the influences of the paternal environment and the impacts of the intergenerational insults on fetal development. ${ }^{4}$ Longitudinal cohort studies that follow participants over time, taking repeated measures, provide compelling data and are powerful research tools to determine associations between a variety of health outcomes and can help drive significant policy change. ${ }^{5}$

Although there have been 17 Australian birth cohort studies so far, only the following two have been federally funded: the Longitudinal Study of Australian Children established in $2004^{6}$ and Footprints in Time: Longitudinal Study of Indigenous Children established in 2008. ${ }^{7}$ Although these two cohorts began the innovative approach of 


\begin{tabular}{|c|c|c|c|}
\hline Cohort name & Status & Australian state or territory & References \\
\hline Port Pirie Cohort Study & $\mathrm{O}$ & SA & 32 \\
\hline Adelaide Nutrition Study & $\mathrm{C}$ & SA & 33 \\
\hline Brunswick Family Study & $\mathrm{C}$ & VIC & 34 \\
\hline $\begin{array}{l}\text { Mater-University of QLD Study of } \\
\text { Pregnancy }\end{array}$ & $\mathrm{O}$ & QLD & 12 \\
\hline The Raine Study & $\mathrm{O}$ & WA & 13 \\
\hline Aboriginal Birth Cohort & $\mathrm{O}$ & NT & 35 \\
\hline Adelaide Respiratory Cohort & $\mathrm{C}$ & SA & 36 \\
\hline $\begin{array}{l}\text { Nepean Study/Nepean Kids Growing } \\
\text { up Study }\end{array}$ & $\mathrm{C}$ & NSW & 36 \\
\hline Peri/Postnatal Epigenetic Study & $\mathrm{O}$ & VIC & 39 \\
\hline $\begin{array}{l}\text { Triple B Study: Bumps, Babies and } \\
\text { Beyond }\end{array}$ & $\mathrm{O}$ & NSW & 40 \\
\hline Watch Study & $\mathrm{O}$ & NSW & 41 \\
\hline Gudaga Study & $\mathrm{O}$ & NSW & 8 \\
\hline Gomeroi gaaynggal & $\mathrm{C}$ & NSW & 42 \\
\hline Splash & $E$ & VIC & 43 \\
\hline VicGen & $E$ & VIC & 44 \\
\hline ORIGINS & $E$ & WA & 45 \\
\hline
\end{tabular}

C, completed; E, emerging; NSW, New South Wales; NT, Northern Territory; O, ongoing; QLD, Queensland; SA, South Australia; TAS, Tasmania; VIC, Victoria; WA, Western Australia.

linking government administrative data with basic physical and cognitive information, these children are now aged 15 and 10 years, respectively, highlighting a need for more recent data to inform policy. As children were the point of interest for each of these cohorts, important antenatal, postnatal and early life data were not collected from parents, and no biological samples were accrued.

A systematic review of longitudinal birth cohorts in Australia that included measures from one parent and offspring is shown in table 1 . Of these cohorts, almost all were established $>20$ years ago, only half of the studies included antenatal measures, while less than half involved the partner. Of studies that included partners, only three studies had biological samples collected from the father. Research involving individuals who identify as First Nations People was minimal and identified only the following two cohorts with specific focus on these groups: the Gudaga cohort in Sydney, New South Wales (NSW) ${ }^{8}$ and the Aboriginal Birth Cohort in the Northern Territory (NT) ${ }^{9}$ However, since the time of this publication there have been others developed in Australia, including the Gomeroi gaaynggal cohort (NSW) ${ }^{10}$ and Pregnancy and Neonatal Diabetes Outcomes in Remote Australia cohort (NT),${ }^{11}$ that are solely focused on the First Nation community.

Of the Australian birth studies, the two largest are the Raine Study (mothers, $\mathrm{n}=2900$; babies, $\mathrm{n}=2868$ and partners, $n=2804$ ) and the Mater-University of Queensland Study of Pregnancy (MUSP; mothers, $\mathrm{n}=7631$; babies, $\mathrm{n}=7223$ and partners, $\mathrm{n}=522$ ) with a retention of $82 \%$ and $53 \%$ at 14 and 21 years, respectively. ${ }^{12} 13$

The Raine Study was established in Western Australia in 1989 with a total of 2900 women birthing in Perth enrolled. ${ }^{13}$ Infants from the cohort have been followed for 27 years. Collection of data began at 18 gestational weeks of pregnancy and has included diverse survey topics of participants including education, toxin exposures, breastfeeding child behaviour, physical activity, mental health from mothers, fathers, offspring, as well as biological samples from the same. Although the investigators of this cohort suggest that this is perhaps the most comprehensive longitudinal study of its kind to date in Australia, there is no doubt that significant societal change has occurred in the 30 years since it began. As such, intrauterine and paternal influences during conception and pregnancy are likely to be markedly different. 
Only two of the studies identified in the systematic review by Townsend et al, ${ }^{14}$ originated in QLD-Environments for Healthy Living (EHL) Griffith birth cohort and the MUSP cohort. The larger of the two (MUSP) was established $>35$ years ago and continues to follow participants with a focus on sociological and psychological outcomes. ${ }^{15}$ Although its impacts on QLD health practice and policy have been considerable, since the inception of this cohort, there have been significant societal changes. The MUSP cohort did not recruit partners, and only $4.04 \%$ ( $\mathrm{n}=273$ from 6527 recruits) were First Nations People with poor retention rates of this subgroup limiting the capacity of the study to fully appreciate the health needs of First Nation families during the perinatal period. ${ }^{15}$ The QLD population has changed significantly since 1980 with a dramatic rise in obesity rates now affecting up to $50 \%$ of the population, ${ }^{16}$ an increase in maternal age at first birth, rising rates of childhood asthma and allergy, ${ }^{17} 18$ a significant change in dietary behaviour, ${ }^{19}$ a rise in immigration and communities with a diverse range of health problems.

The EHL Griffith birth cohort began in Logan, Gold Coast and Tweed regions of south-east QLD and Northern NSW in 2006. ${ }^{20}$ Unlike many other cohorts in Australia, this cohort included an investigation of social, environmental, neighbourhood and family functioning within its DOHaD-style cohort. Cord blood was also collected. This cohort was designed in partnership with another research consortium in Wales, UK so that the EHL Wales cohort could provide international comparisons. Data linkages for Medicare Benefits Scheme (MBS), Pharmaceutical Benefits Scheme (PBS) and the child's immunisation history were obtained from the Medicare records. Maternal perinatal history was obtained from health records. The EHL Griffith birth cohort ceased recruitment and follow-up of participants several years ago when funding for the study ceased.

Looking to the future of birth cohorts in QLD, the Mater Hospital in Brisbane is in a strong position to establish such a cohort particularly given it has the highest birth rate in QLD $^{21}$ and provides tertiary and quaternary level health services for both high, and low socioeconomic status (SES) populations. Recently, the Queensland Family Cohort (QFC) completed a pilot feasibility study in Brisbane. The protocol for the QFC study was developed through a consultation process with QLD health researchers with expertise in DOHaD methodology. This multistage process resulted in a series of 21 research themes for the cohort. Each theme has its own series of questions and the methodologies to answer these that have been developed by a collaborative group.

The particular strengths of this cohort design are its (1) recruitment from entire state of QLD ( 1.85 million $\mathrm{km}^{2}$ ), which has a population of 5.2 million including approximately $4.3 \%$ First Nation community members; (2) detailed collection of physical, social and mental health data of mother, father/partner and child; (3) biological samples collected at multiple time points from all family members; (4) all survey instruments are based on validated tools and routinely collected clinical data; (5) inclusion of biological samples were agreed by the research collective only after detailed justification by individual researchers; (6) recognising the equal importance of the family relationships on the child so that parents and partners are all involved; (7) inclusive of same sex relationships and single parents and (8) inclusion of any participant regardless of chronic and acute health conditions.

The data obtained will enable characterisation of all participants' health and social experiences for each family unit enrolled in the study to identify the current trends in health and health behaviours. It will identify immediate and future health requirements of the QLD population which will have a significant impact on health policy and practice and help define preventative interventions required for the health of future generations of Australians. Using this rich data source, we will endeavour to understand the biological mechanisms that may contribute to adverse health outcomes in parents and their children. This will drive future interventions in healthcare that include lifestyle, pharmacological and clinical modifications in practice.

\section{METHODS AND ANALYSIS}

\section{Study design and setting}

The QFC is a prospective, observational, longitudinal study that will be piloted at the Mater Mothers' Hospital (MMH) from 2018 to 2021 with an aim to continue for three decades. Families will be followed throughout pregnancy, childbirth and infancy via individually funded, separate follow-up studies. Long-term follow-up of these participants and their children via data linkage is the aim. Although participant numbers for the pilot study have been attained, the research team continued to recruit into the cohort when COVID-19 pandemic arose to further understand implications of COVID-19 on families during the pandemic, although with a modified protocol.

Following thorough analysis of the pilot data, additional sites will be added across QLD after 2021, and will be inclusive of metropolitan, rural and remote communities. Additionally, this study will partner with First Nation communities and organisations to modify protocol methods to ensure the inclusion of these community members.

\section{Inclusion criteria}

For the pilot phase of the study, pregnant individuals who are booked to deliver at the MMH will be eligible to participate. Following the pilot project, however, this will extend into a multisite programme across QLD health services. Pregnant individuals who are permanent residents of Australia and reside in QLD, are 12-24 weeks pregnant and their partners, if they have one, will be invited to participate in the pilot study. To ensure the cohort is representative of the QLD population, every effort will be made to invite all eligible individuals to 
participate in the study including those from non-English speaking backgrounds, $<18$ years of age, with special needs and First Nation community members. Importantly, this cohort is seeking a true understanding of the breadth of health issues for families, so that participants with any underlying serious or chronic health conditions will be eligible for inclusion in the study.

\section{Exclusion criteria}

As this cohort has a significant focus on the health of the family unit, if the pregnant person would like to participate in the cohort, but their partner does not consent, or vice versa, then they will be excluded from the study. If either person is unable to give informed consent, they will be excluded from participating in the study.

\section{Recruitment and consent}

Potential pregnant participants and their partners will be approached for participation in the study by suitably qualified experienced research midwives either face-to-face, via phone or via SMS. Alternatively, potential families can also contact the study via publicity generated by social media and marketing campaigns detailing the QFC study. Regardless of the mode used to make initial contact, those interested in participating will be provided with the participant information statement prior to obtaining written consent.

\section{Study regimen}

Active data are collected from pregnant participants and their partners throughout their pregnancy, delivery and into early infancy, as shown in table 2. Active data collection will continue in multiple locations in QLD up to 3 years gestational age corrected, with lifelong, passive data collection via MBS and PBS data linkage.

For the pilot study, data will be collected four times during pregnancy (12-24, 24, 28 and 36 weeks), at delivery, postdelivery (on the ward) and at 6 weeks postpartum. Table 2 outlines items collected at each of these occasions that will be undertaken by the participant unaided, while table 3 highlights the time and those items collected with the assistance of a research team member. In brief, these will be collected as follows: 12-24 weeksconsent and enrolment forms completed by pregnant participant and their partner; 24weeks-is a study visit for pregnant participant and partner for questionnaires, biological sample collection from both and an ultrasound growth scan; 28 and 36 weeks — each has a study visit for pregnant participant only with questionnaires, biological samples and a ultrasound growth scan collected; deliveryplacenta, cord blood sample collection and chart review; on-ward visit-chart review, questionnaire and biological samples collected from mother and baby; 6 weeks postpartum - breastfeeding assessment, body composition of infant, questionnaires and biological sample collection from mother. Consent for data linkage will be sought for participants from the QFC pilot study.
Study data will be collected and managed using Research Electronic Data Capture (REDCap) electronic data capture tools hosted at University of Queensland..$^{22} 23$ REDCap is a secure, web-based software platform designed to support data capture for research studies providing (1) modifiable user rights to protect identifiable data; (2) an intuitive interface for validated data capture; (3) audit trails for tracking data manipulation and export procedures; (4) automated export procedures for seamless data downloads to common statistical packages and (5) procedures for data integration and interoperability with external sources. ${ }^{23}$ Data collected will be entered into REDCap by a research nurse/midwife, research assistant or another suitably trained member of the research team. Double data entry and cross validation methods will be used to ensure validity and quality of data.

The QFC study has focused on data collection and outcomes from 21 major themes from the QFC research collaborative (figure 1), each with their own series of study questions highlighted in online supplemental table 1. Data collected across the themes included participant questionnaires and routinely collected clinical data from medical charts (demographics, medication usage, medical history, pathology, medical imaging and other clinical results), as well as intervention data (biological samples, physical and psychological assessments obtained for research purposes only). Data will be collected by experienced and qualified research nurses, midwives and research assistants. Some data will be collected by research-trained allied health professionals, including sonographers, dieticians, physiotherapists and psychologists prospectively from patient notes, observation charts, study specific questionnaires and assessments, photographs and videos, and electronic laboratory results by research staff and the principal investigator. Data collection forms will be electronic, except in cases where the participant does not have reliable access to a computer or the internet, or where a participant requests the use of paper forms.

\section{Primary outcomes}

This cohort has the following three primary outcomes to determine: (1) current status of parental physical and mental health and its impact on health of offspring; (2) explore genetic and biological mechanisms that drive chronic disease risk in pregnancy, in childhood and in later life and (3) understand the influence of the environment and climate change on the health of future generations.

\section{Assessments}

The associated measures, assessments and sample collections for the cohort are listed in tables 2 and 3. All questionnaires for the study have been either used in routine clinical care or previously validated and references are included within the tables where appropriate. Secondary outcomes specific to the individual themes are listed in online supplemental table 1. 


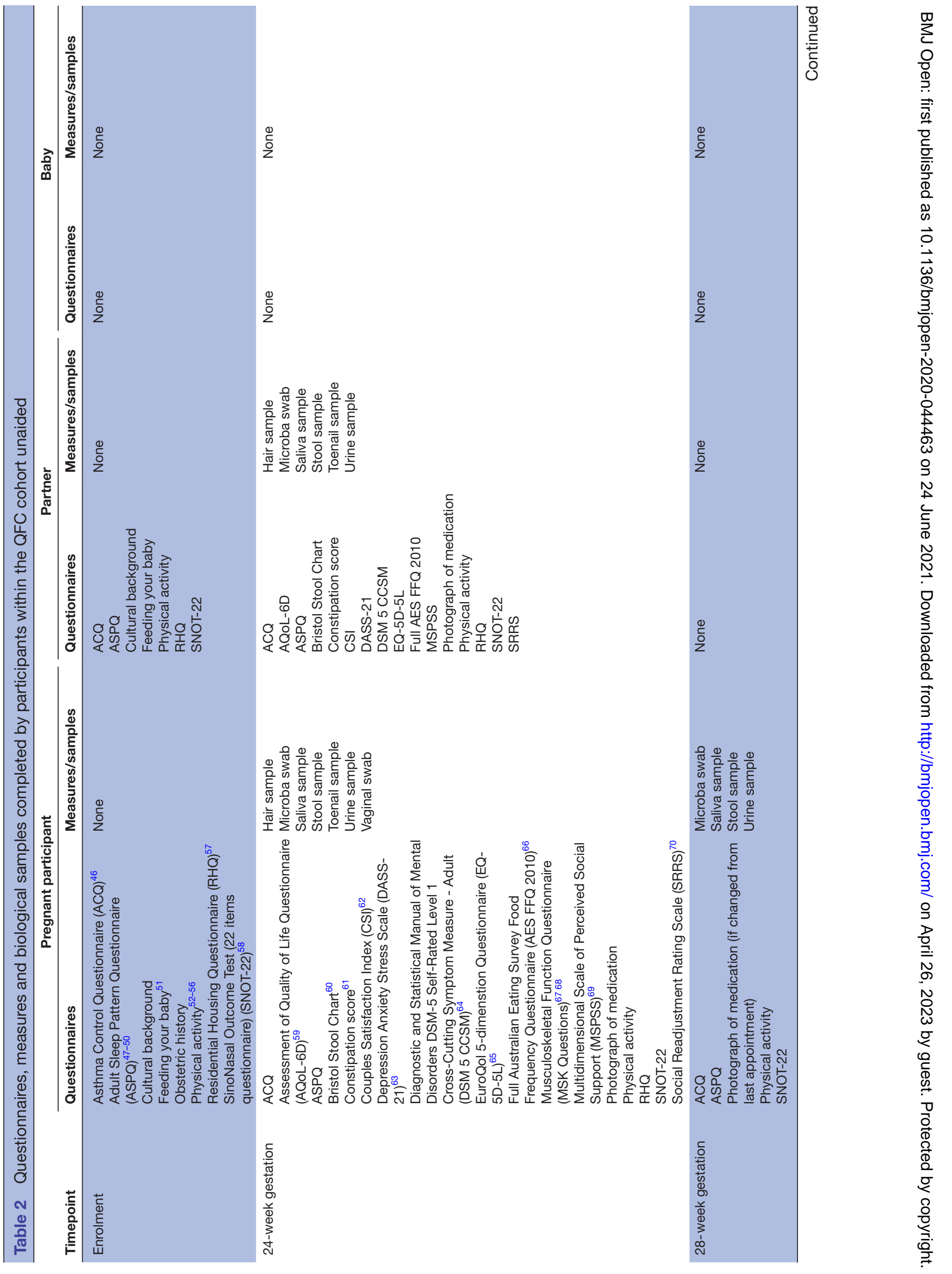




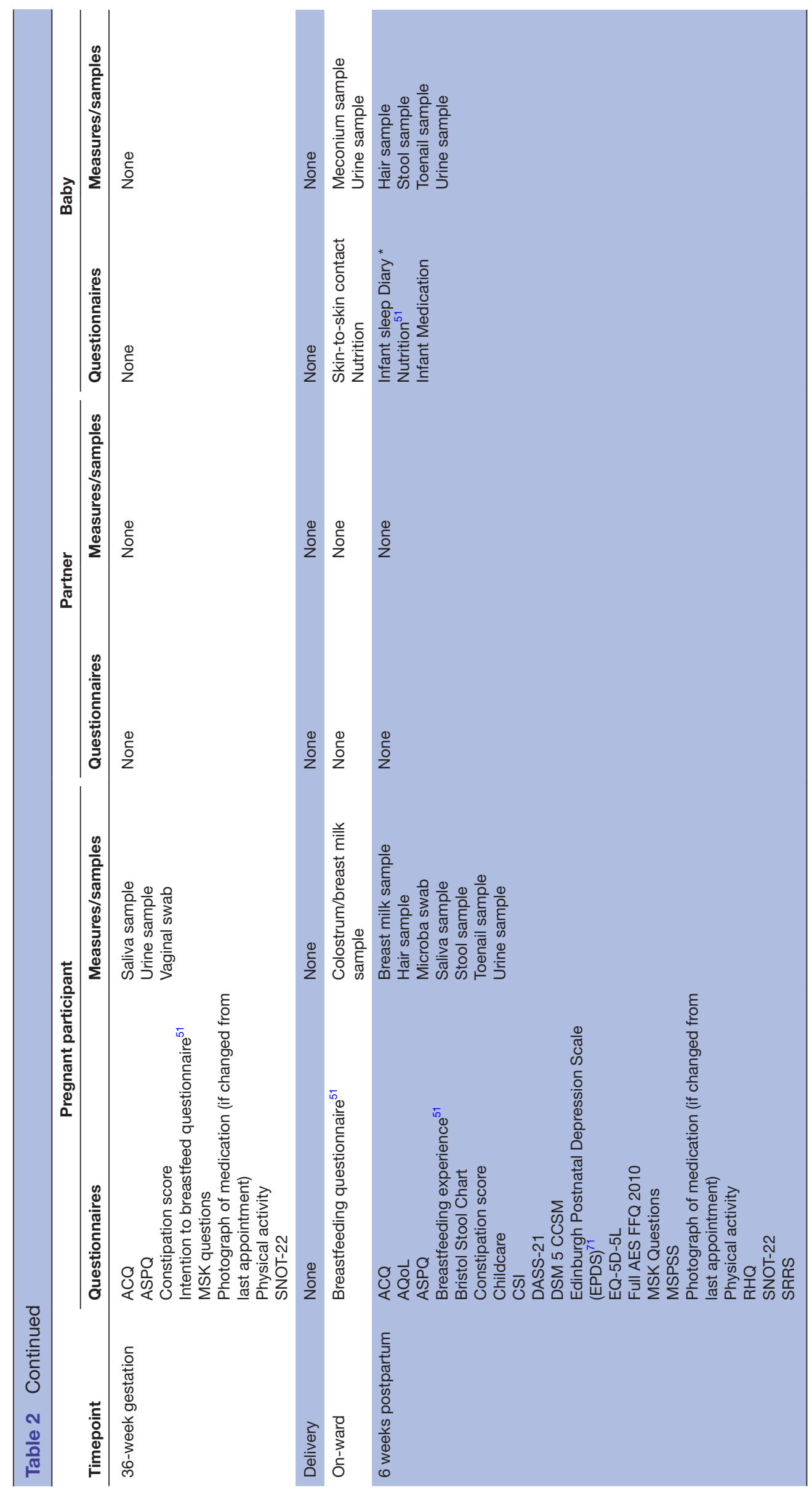

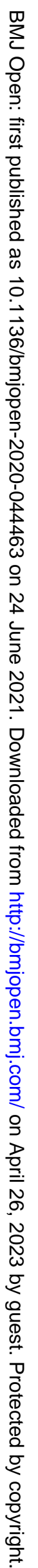




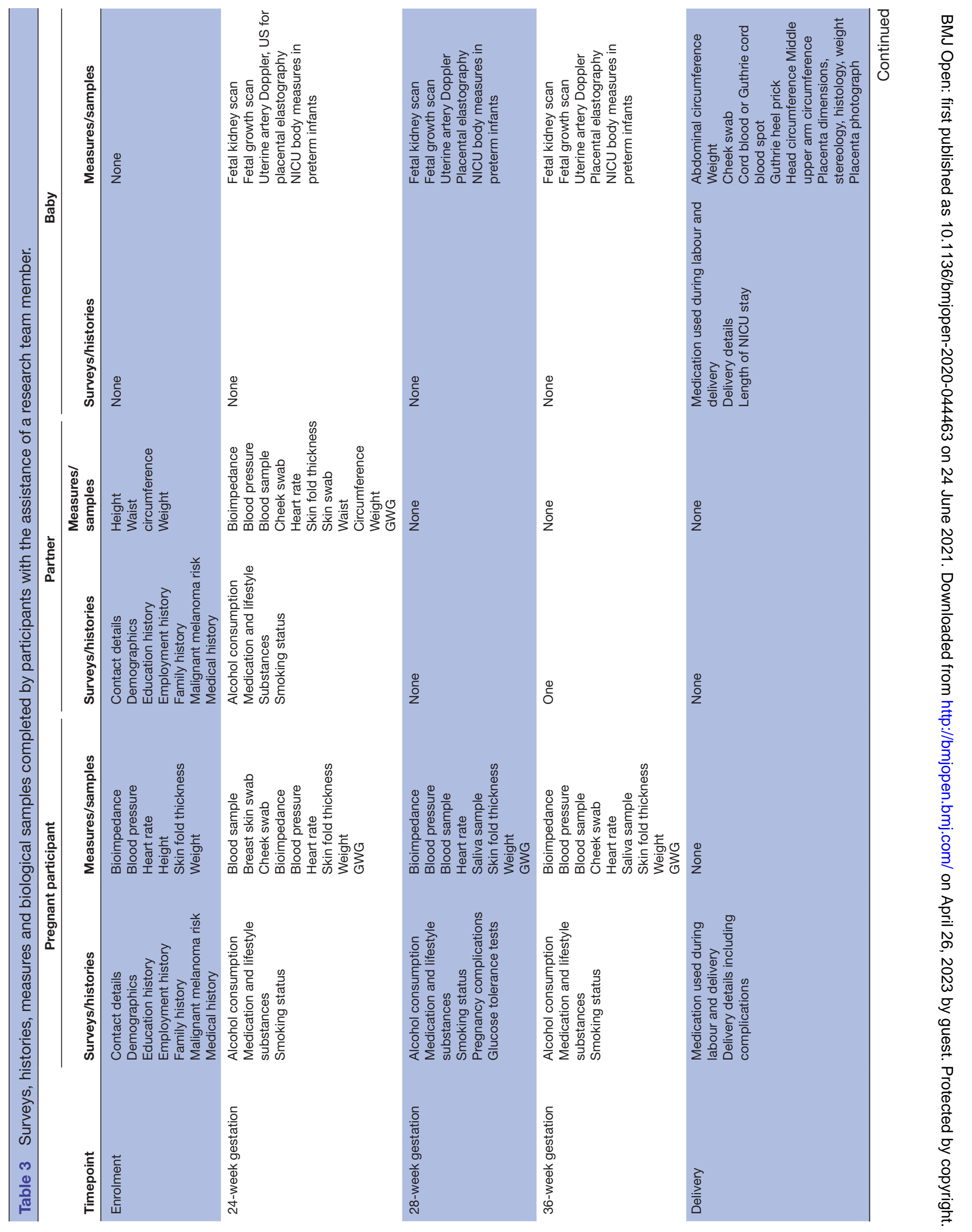




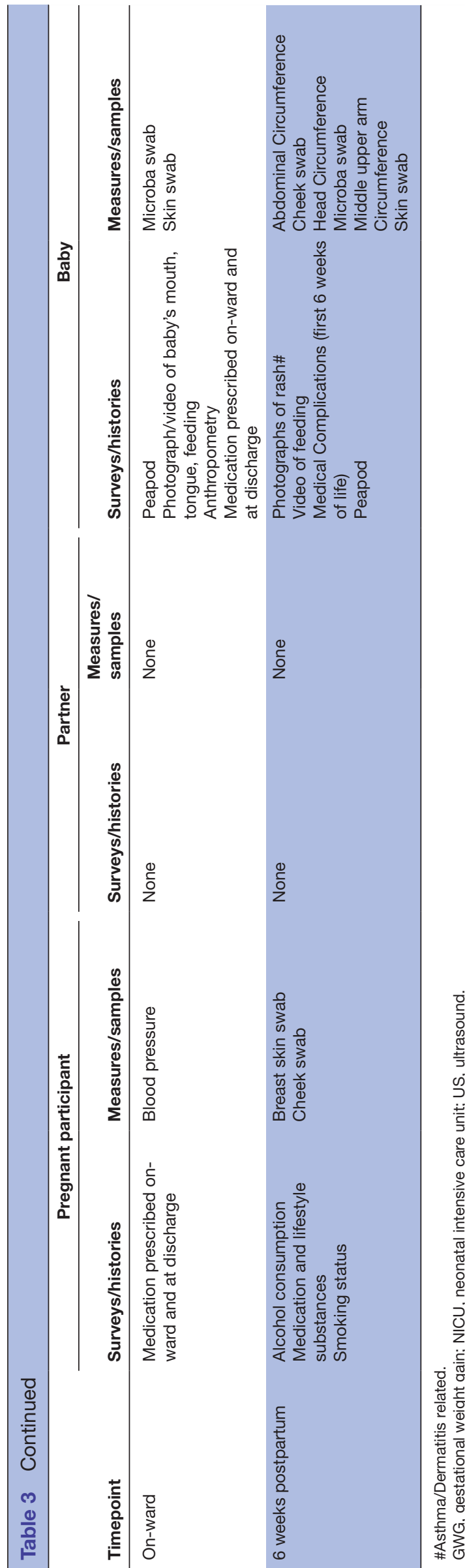

\section{Sample size}

A sample size of 12500 QLD families was chosen so that some of the rarer complications of pregnancy can be interrogated with sufficient statistical power. For example, delivery of small-for-gestational infants occurs in approximately 6.7\% QLD deliveries and a stillbirth delivery occurs in approximately $0.6 \%$ of QLD deliveries, thus warranting a large cohort size. ${ }^{24}$

\section{Statistical analysis}

Missing outcome data will not be imputed in the primary analysis, as the key assumption of missing at random is not likely to hold in the analysis population. Sensitivity analyses will be conducted, however, using the multiple imputations method to explore the potential impact of missing data on outcomes. The characteristics of those participants with missing data will be compared between two treatment groups (healthy vs unhealthy outcomes). Baseline characteristics of babies and their parents will be summarised for each site, as well as overall, using descriptive statistics. Continuous variables will be reported as numbers of observed and missing values, mean, SD, median and range. Categorical variables will be described as frequencies and percentages.

\section{DATA AVAILABILITY STATEMENT}

Data from the QFC study will be made available in the future for collaborative research questions. Such requests must be authorised by the principal investigators, the QFC Research Governance Committee and the appropriate Human Research Ethics Committees and Human Research Governance Safety Entities.

\section{PATIENT AND PUBLIC INVOLVEMENT}

The protocol for the pilot study was developed with extensive engagement within the QFC Research Collaborative community and a community engagement group including consumers (individuals aged 18-65 years). This group highlighted that understanding mental health and social support networks were essential for a vulnerable population such as pregnant women (and their families). Accordingly, both mental and physical well-being are strong components within the QFC study design (table 2). In addition, the QFC Research Collaborative compromised of research academics and clinicians (figure 1), identified the clinical data, biological samples and questionnaires necessary for taking a snapshot of a reproductive age population that would also capture the consumers suggestions raised in the community engagement group.

The QFC team are currently working in partnership with the First Nation communities of QLD to determine how to best ensure that this protocol is truly inclusive of their community members. At the time of writing, the research team developed the terms of reference to establish a new consumer panel who are either 


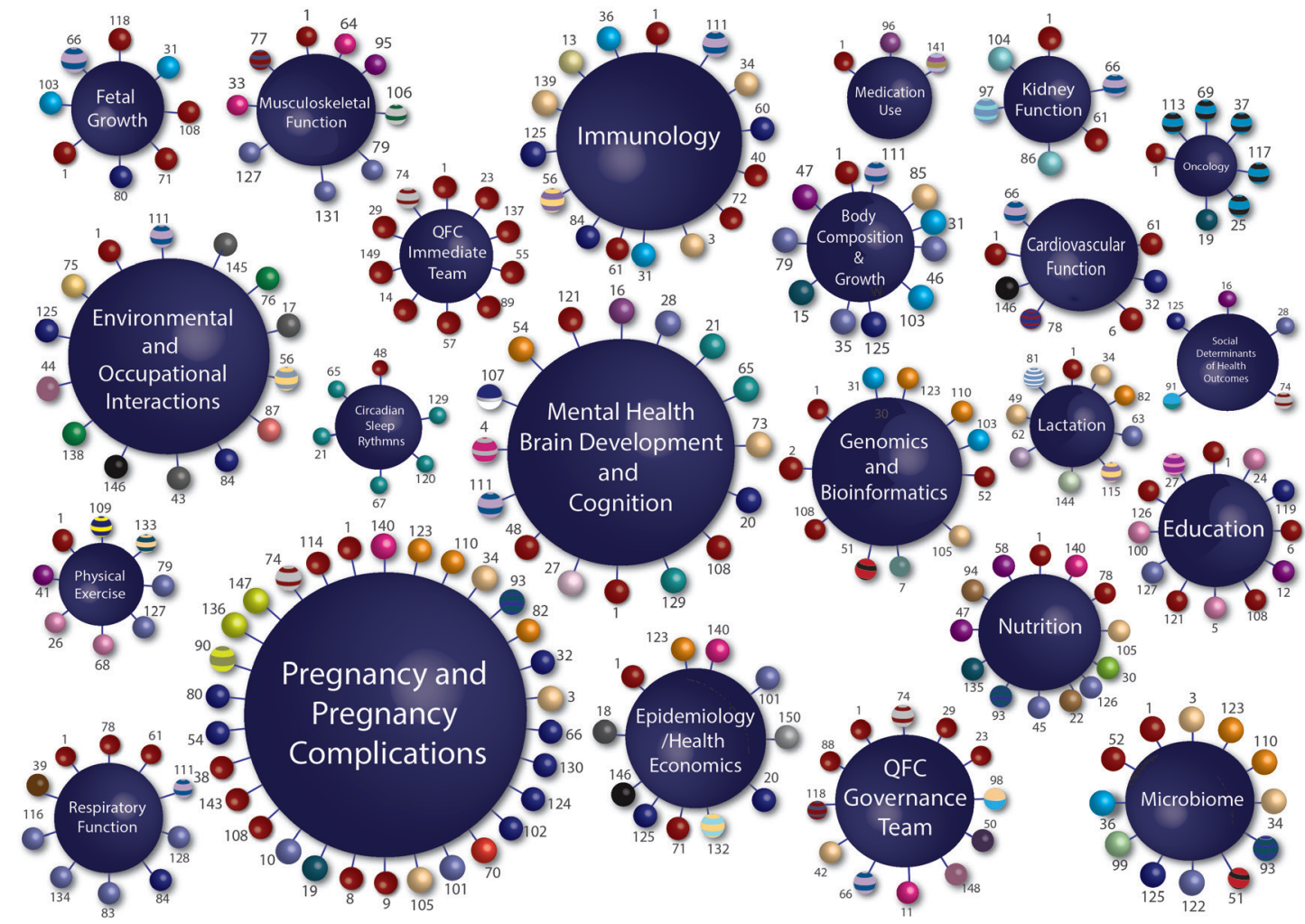

Figure 1 The QFC research collaborative. Each large sphere (navy blue) represents one of the 21 research themes that compromise the QFC research collaborative. Each number represents an individual researcher (150 researchers to date). Each small sphere represents the affiliation of that particular researcher (51 collaborating institutes/universities). These affiliation areas follows: ACU (Faculty of Health Sciences); ACU (Institute for Learning Science and Teacher Education); ACU/ UQ (Faculty of Health and Behavioural Sciences); Child Health Research Centre; Griffith University; James Cook University (Australian Institute of Tropical Health \& Medicine); James Cook University (Department of Medicine); Mater Health; Mater Health Pathology(consultant microbiologist); Mater Health/Princess Alexander Hospital; MaterHealth/ Queensland Children's Hospital; Mater Research-UQ/Mater Health; Mater Research/Carbal MedicalServices; Mater Research-UQ; Menzies Health Institute Queensland/Griffith University (Griffith Health); Metro South Health/QUT (Faculty of Health); Metro South Health/ Princess Alexander Hospital; Monash University (Faculty of Medicine, Nursing and Health Sciences); NSW Department of Planning, Industry and Environment; Pathology Queensland; QIMR Berghofer; QUT (Faculty of Health); QUT (Faculty of Health)/ Child Health Research Centre; QUT (Faculty of Science and Engineering); QUT (Faculty of Science and Engineering)/Child Health Research Centre; QUT (Faculty of Health)/UQ (Centre of Clinical Research); QUT/University ofGeorgia; South Australian Health and Medical Research Institute; Sun Yat-SenUniversity; University of Newcastle; University of Southampton; UQ (Institute for Social Science Research; Faculty of Humanities and Social Sciences); UQ(Centre of Clinical Research); UQ (Diamantina Institute); UQ (Diamantina Institute)/Dermatology Research Centre; UQ (Discipline of General Practice;Primary Care Clinical Unit); UQ (Faculty of Business, Economics \& Law); UQ (Faculty of Engineering, Architecture \& Information Technology); UQ (Faculty of Health and Behavioural Sciences); UQ (Faculty of Health and Behavioural Sciences)/Bond University (Faculty of Health Sciences \&Medicine)/Mater Health; UQ (Faculty of Medicine); UQ (Faculty of Medicine)/Child Health Research Centre; UQ (Faculty of Medicine)/Centre for Health Services Research; UQ (Faculty of Medicine)/Mater Research-UQ; UQ (Faculty of Medicine)/Perinatal Research Centre; UQ (Faculty of Science)/UQ (Centre of Clinical Research); UQ (Institute for Molecular Bioscience); UQ (Institute forSocial Science Research); UQ (Queensland Brain Institute); Wesley Hospital Monash IVF and Wesley Hospital Monash IVF/University of Melbourne.

expecting a baby; have young children or are QFC pilot participants, who will further refine the protocol design from their previous experience and preferences, for the future multisite QFC study. The QFG team will ensure that this group includes partners, and First Nation families. QFC pilot participants were not involved in the recruitment of the study but have provided information in the form of feedback surveys to further refine the study protocol. Lay reports will be made available to interested participants in the form of a newsletter sent to them via email and via formal invitation to a seminar day where researchers will describe individual findings.

\section{SOURCES OF BIAS}

One of the significant sources of bias is related to consent. Under the ethics approval given for the study, the research team can only approach potential participants to join the study if they have indicated that they are willing to be involved in 'any research that is undertaken 
by 'Mater Health' when they complete their antenatal enrolment forms. If the potential participant leaves this blank or indicates no, they cannot be contacted. This is likely to mean that those joining the study are coming from a pool of potential participants that may be better educated and of higher SES. ${ }^{25}$ Although all potential pilot participants will be approached and followed-up using phone calls or SMS reminders, selection bias may be introduced for potential participants who are linguistically diverse and may fail to respond to follow-up attempts. ${ }^{26}{ }^{27}$ Moreover, while we acknowledge that understanding the reason and demographics of families who do not consent to participant in this study would be of benefit, under the ethics approval for the pilot study, we are unable to follow-up or question a participant once they refuse contact. Like many other cohort studies, this protocol relies on the collection of self-reported data from participants. This is an inherent source of bias in self-collected data. ${ }^{28}$

\section{ETHICS AND REGULATORY ASPECTS \\ Ethics}

The QFC study has been approved by the Mater Misericordia Limited Human Research Ethics and Governance Safety Committee (HREC/16/MHS/113).

\section{Participant safety}

All risks to the participants in the study will be mitigated by ensuring all recruitment and data collection is managed by appropriately trained and experienced research staff which have had training in empathetic, cross-cultural communication. Recruitment staff will be research nurses and midwives with extensive experience in research and clinical practice. Data will be stored in a secure setting and data linkage software will be adequately protected to maintain security and privacy.

\section{Study governance}

The QFC study is overseen by the principal investigators and the QFC Research Governance Management Committee. This committee will meet every 2 months to (1) continue to develop the strategic directions and priorities for the protocol; (2) review all substudy applications to the cohort prior to their submission to ethics and (3) review all unexpected findings from the analysis under an ethically defensible plan with the QFC Clinical Advisory Panel ${ }^{29}$ Each QFC research theme has a theme leader who will report annually and develop the individual research hypotheses within their theme. New research questions require a substudy application that outlines what data requirements the applicant requires for their application along with their planned analysis. When approved by the QFC Research Governance Management Committee, the substudy application and reference letter from the QFC Research Governance Committee is attached as supporting documents to a new Human Research Ethics application, where approval is sought. Most recently, the
QFC Research Governance Management Committee has identified that a community-based Consumer Advisory Committee would be of benefit to the long-term development of the cohort as it continues into the future. The terms of reference for this group have been developed and its establishment is planned for late 2021.

\section{Unexpected findings during examinations}

Participants are advised during the consenting process that if disclosures are required by law, or there are health concerns during routine appointments that require urgent care, that it is the duty of care of the research midwives to disclose or escalate the issue as appropriate. If analysed data from the study were to reveal findings that bear on the well-being of participants, their relatives or their community, whether anticipated or incidental to the scope of the research, the QFC Research Governance Committee team will be formally notified. The QFC Research Governance Committee will create a panel of experts to cross-check research findings for accuracy (QFC Clinical Advisory Panel). Only those confirmed research findings that are clinically actionable, where there are established therapeutic or preventative interventions or other available actions, such as lifestyle changes or reproductive decisions, that have the potential to change the clinical course of the disease or improve the individual's and/or their genetic relative's quality of life, will be considered to be returnable to the participant. This panel will, in conjunction with representatives from the QFC Research Governance Committee, identify and recontact the participant initially via letter, and then by telephone. $^{29}$

\section{Dissemination}

The findings from the analysis of cohort data will be disseminated in a variety of ways including abstracts, posters and presentations at conferences and published manuscripts in peer-reviewed journals. These will also be reported to federal, state and local governments to inform policy and reports made to funding bodies, institutes and hospitals that participated in and supported the cohort study. Members of the study team will have publishing and authorship rights in accordance with National Health and Medical Research Council Australian Code for the Responsible Conduct of Research, the International Committee of Medical Journal Editors requirements for authorship, and as described in research agreements.

\section{DISCUSSION}

The QFC is of enormous significance to Australia, particularly to the state of QLD. The only previous longitudinal cohort established in QLD was initiated $>30$ years ago, ${ }^{12}$ and while its contributions continue to impact on policy, this cohort sampled only within metropolitan Brisbane and failed to include rural and remote QLD communities. Since its inception, there have been significant societal changes including an increasing burden of 
chronic disease in adulthood, thus impacting the health outcomes of pregnancy and infancy. Additionally, the impact of other influences such as home environment, ${ }^{30}$ social determinants of health ${ }^{31}$ and the role of fathers ${ }^{4}$ on the child are becoming better understood, and the QFC is one of the few Australian cohorts that includes direct sampling of partners. This work will lead to identification of immediate and future health requirements of Australian families, and a solid body of evidence with which to develop well-defined intervention studies to improve health.

The unique outcomes of the QFC will include the following: (1) deep analysis of maternal and paternal health during antenatal and postpartum periods; (2) examination of perinatal outcomes; (3) life-course analysis of maternal, paternal and infant health outcomes; (4) analysis of biological samples to understand biological mechanisms driving health outcomes and (5) development of rich data that characterises health, social, environmental and educational experiences of family members that can lead to improve understanding of health and disease for all family members during this period. To our knowledge, there is no other cohort within Australia and very few internationally, where all these five points are incorporated into a single study cohort.

The QFC pilot study has so far demonstrated the ability to recruit pregnant participants from private and public sectors, including specialised clinics such as diabetes and refugee clinics. Participating families had individual members born in 53 different countries including Australia, with the maternal age of conception spanning 24 years. Furthermore, our families have identified as single (pregnant participant only), cis-gender and LGBTQIA+ (lesbian, gay, bisexual, transgender, queer, intersex and asexual) members. Overall, there has been a $10.6 \%$ withdrawal rate by 6 weeks postpartum. This suggests while still ongoing, the QFC study has the ability to cover the entire range of socioeconomic and cultural groups within the QLD population.

Like many longitudinal cohort studies, ${ }^{14}$ a significant limitation of the project is the cost of establishing and maintaining a cohort over a number of decades, storing its biological samples and the costs of analysis. To overcome these limitations, the principal investigator has developed this as a multi-institutional initiative that encompasses the entire state, with each institute contributing financially or in-kind towards the cohort (figure 1). Additionally, the study protocol has not yet completed cultural consultation with the First Nations communities although it is in process with in-principal agreements from First Nation organisations as the first outcome achieved. At this point in the study, it is worth noting that the piloted participating families have been recruited in the metropolitan community of Brisbane and its findings, when reported, may not be widely applicable in rural and remote communities. In conclusion, despite the limitations, analysis of data arising from this cohort will influence policy and practice for healthcare that is based on the current health of QLD families, and provide further understanding of the impact of current health of family members on the health of the next generation.

Author affiliations

${ }^{1}$ Mother and Baby, Mater Medical Research Institute, South Brisbane, Queensland, Australia

${ }^{2}$ Faculty of Medicine, The University of Queensland, Saint Lucia, Queensland, Australia

${ }^{3}$ Gallipoli Medical Research Foundation, Greenslopes, Queensland, Australia ${ }^{4}$ Maternity Unit, Greenslopes Private Hospital, Greenslopes, Queensland, Australia ${ }^{5}$ School of Clinical Sciences, Faculty of Health, Queensland University of Technology, Brisbane, Queensland, Australia

${ }^{6}$ Centre for Maternal Fetal Medicine, Mater Hospital Brisbane, Brisbane, Queensland, Australia

${ }^{7}$ Institute of Health \& Biomedical Innovation, Queensland University of Technology, Brisbane, Queensland, Australia

${ }^{8}$ School of Medical Science, Griffith University - Gold Coast Campus, Southport, Queensland, Australia

${ }^{9}$ The University of Queensland Child Health Research Centre, South Brisbane, Queensland, Australia

Twitter Danielle Borg @DrDanBorg and Christopher Edwards @edchris_

Acknowledgements We acknowledge the Yuggera People as the traditional custodians of the land in which the pilot study was conducted. We pay respect to the elders of this community, past, present and emerging. We acknowledge the families who agreed to participate in pilot stages of this cohort and look forward to their continued involvement as their family grows. We also acknowledge the contributions of the Mater Mother's Hospital, the theatre nurses, midwives in delivery and postnatal wards, Mater pathology staff, the placenta collection team who assisted in collection of samples when required. We thank Dr Mike Beckmann and Mater Research Development, Ethics, Governance and Agreements departments for the ongoing support. We thank the staff at Microba and Qiagen for their ongoing collaboration. We acknowledge the researchers and institutes who form the QFC Research Collaborative which helped support the QFC study. Finally, we thank Dr Aimee Catalan of Catalan Group Ltd Pty for the design and production of figure 1.

Collaborators The following are members of the Queensland Family Cohort Research Collaborative and have all been integral to the overall design of the study: Adam Ewing (Mater Research Institute-UQ (MRI-UQ)), Alison Carey (QLD University of Technology (QUT)), Amanda Wheeler (Menzies Institute of Health Griffith University), Ameneh Shahaeian (Australian Catholic University (ACU)), Andrew P Hills (MRI-UQ), Andrew Perkins (Monash University), Ann Peacock (MRI-UQ), Anne Tremellen (MRI-UQ), Annie McArdle (Mater Health), Anthony Tuckett (University of QLD (UQ)), Asad Ali (UQ), Ash Meakin (MRI-UQ), Barbara Lingwood (UQ), Barnaby Dixson (UQ), Boyi Yang (Sun Yat-Sen University), Brenda Gannon (UQ), Carlos Salomon (UQ), Caroline Salom (UQ), Cassandra Pattinson (UQ), Clare Collins (University of Newcastle (UON)), Claire Wyatt-Smith (ACU), Clare Primiero (UQ), Courtney Giles (ACU), Cynthia Turner (ACU), Daniel Schweitzer (Mater Health), Danielle Schoenaker (University of Southampton), David Evans (UQ), David Simmons (UQ), Dilani Mendis (Griffith University (GU)), Elise Pelzer (QUT), Elizabeth Hurrion (Mater Health), Emma Hamilton-Williams (UQ), Erin McMeniman (UQ), Frances Maguire (MRI-UQ), Geraint Rogers (South Australian Health and Medical Research Institute), Greg Monteith (MRI-UQ), Gregore Iven Mielke (UQ), Guang Hui Dong (Sun Yat-Sen University), Gunther Paul (James Cook University (JCU)), Helen Barrett (Mater Health), Helen Liley (Mater Health), Helen Truby (UQ), Honey Heussler (MRIUQ), Honor Hugo (QUT), lan Wright (JCU), Jake Gratten (UQ), Jakob Begun (MRI-UQ), James Cuffe (UQ), James Scott (QLD Institute of Medical Research-Berghofer (QIMR-B)), Janet Davies (QUT), John Cairney (UQ), John Hooper (MRI-UQ), John Upham (UQ), Josephine Forbes (MRI-UQ), Julianne McGuire (QUT), Julie Germain (Mater Health), Julie Hides (GU), Kalina Rossa (UQ), Karen Thorpe (UQ), Kassia Beetham (ACU), Katie Lee (ACU), Kerry Richard (Pathology QLD), Kristen Gibbons (MRI-UQ), Kristen Radford (MRI-UQ), Kristin Laurens (QUT), Leisa-Maree Toms (QUT), Lidia Morawska (QUT), Liisa Laasko (Mater Health), Linda Gallo (UQ), Linda Hickey (MRI-UQ), Lisa Akison (UQ), Loretta Anderson (Mater Health), Lucia Colodro-Conde (QLD Institute of Medical Research-Berghofer), Lucy Burr (Mater Health), Luke Knibbs (UQ), Lynne Daniels (QUT), Magid Fahim (Princess Alexander Hospital, QLD), Mandana Mazerheri (NSW Department of Planning, Industry and Environment), Maree Knight (MRI-UQ), Mark Green (Wesley Hospital Monash IVF), Mark Western 
(UQ), Marloes Dekker (UQ), Megan Rollo (UON), Melinda Smith (UQ), Meng-Wong Taing (UQ), Micheal Burke (Mater Hospital), Micheal Kimlin (QUT), Micheal Thomas (Mater Health Pathology), Michele Haynes (ACU), Mike Beckmann (Mater Health), Natasha Reid (UQ), Nicole Warrington (UQ), Nikky Isbel (Metro South Health, QLD), Olivia Holland (QUT), Paige Little (QUT), Paul Colditz (UQ), Paul Dawson (MRI-UQ), Paul Gardiner (UQ), Penelope Lind (QIMR-B), Peter D. Sly (UQ), Peter Soyer (UQ), Rebecca Byrne (QUT), Rebecca Keating (Mater Health), Rick Sturm (UQ), Sally Schaffer (UQ), Samudragupta Bora (MRI-UQ), Sanmarie Schlebusch (Mater Health), Sarah Medland (QIMR-B), Sarah Steane (UQ), Shamshad Karatela (UQ), Shelley Wilkinson (MRI-UQ), Sheridan Guyatt (Mater Health), Simon Bowler (Mater Health), Simon Smith (UQ) Sophia Yong (UQ), Steve Erceg (Mater Health), Steve McPhail (Metro South Health QLD), Stewart Trost (UQ), Suresh Sandisivam (Mater Health), Susan de Jersey (UQ), Tara Ross (Wesley Hospital Monash IVF), Tom Cole-Hunter (QUT), Tony Kenna (QUT), Treasure McGuire (UQ), Vicki Flenady (MRI-UQ), Wendy Brodribb (UQ), Xiao-Wen Zeng (Sun Yat-Sen University), Yang Peng (UQ), Yanhe Liu (Wesley Hospital Monash IVF), Yoga Kandasamy (JCU), Zarqa Said (MRI-UQ) and Zephanie Tyack (UQ).

Contributors DB and KR jointly wrote the manuscript; DB, KR, SK and VC: designed the QFC protocol; CT, TH, JJ-M, FF and MO: responsible for recruitment and retention of participants for QFC; DB, VC, CF, JS, JJ-M, CT, MO and TH: designed the REDCap system for QFC data management; EC, CE, DF and SK: designed ultrasound data collection; EC and CE: collected ultrasound data from QFC participants; DB, CF and JS: designed FreezerPro system for management of QFC participants; DB KR, CT, TP, GdZ, KM, SK and VC: members of QFC Governance committee; CF, JS, JJ-M, CT, MO, TH, EC, CE, DF, TP, GdZ, KM, SK and VC: all reviewed and edited of manuscript on behalf of the Queensland Family Cohort Research Collaborative. All members of the QLD Family Cohort Research Collaboration were involved in establishing the aims and the design of the cohort study.

Funding This study has received cash and in-kind funding from the following organisations and institutes: Mater Foundation (NA); University of Queensland (NA); Griffith University (NA); Queensland University of Technology (NA); Brisbane Diamantina Health Partners (grant no. 1033); The Lott, Golden Casket (grant no. 75002_GC_20); Perpetual Impact Funding (grant no. 2041); Advanced Queensland (grant no. 2441); Qiagen (NA) and Microba (NA).

Competing interests None declared.

Patient consent for publication Not required.

Provenance and peer review Not commissioned; externally peer reviewed.

Supplemental material This content has been supplied by the author(s). It has not been vetted by BMJ Publishing Group Limited (BMJ) and may not have been peer-reviewed. Any opinions or recommendations discussed are solely those of the author(s) and are not endorsed by BMJ. BMJ disclaims all liability and responsibility arising from any reliance placed on the content. Where the content includes any translated material, BMJ does not warrant the accuracy and reliability of the translations (including but not limited to local regulations, clinical guidelines, terminology, drug names and drug dosages), and is not responsible for any error and/or omissions arising from translation and adaptation or otherwise.

Open access This is an open access article distributed in accordance with the Creative Commons Attribution Non Commercial (CC BY-NC 4.0) license, which permits others to distribute, remix, adapt, build upon this work non-commercially, and license their derivative works on different terms, provided the original work is properly cited, appropriate credit is given, any changes made indicated, and the use is non-commercial. See: http://creativecommons.org/licenses/by-nc/4.0/.

ORCID iDs

Danielle Borg http://orcid.org/0000-0001-6126-7583

Sailesh Kumar http://orcid.org/0000-0003-0832-4811

\section{REFERENCES}

1 Barker DJP. Developmental origins of adult health and disease. $J$ Epidemiol Community Health 2004;58:114-5.

2 Gluckman PD, Hanson MA, Cooper C, et al. Effect of in utero and early-life conditions on adult health and disease. N Engl J Med 2008;359:61-73.

3 Gluckman PD, Hanson MA, Pinal C. The developmental origins of adult disease. Matern Child Nutr 2005;1:130-41.

4 Braun JM, Messerlian C, Hauser R. Fathers matter: why it's time to consider the impact of paternal environmental exposures on children's health. Curr Epidemiol Rep 2017;4:46-55.
5 Western M, Foelz C. The case for a new Australian birth cohort study. Brisbane: Institute for Social Science Research, University of Queensland, 2014.

6 Gray M. Growing up in Australia: the longitudinal study of Australian children, 2005: 4-9.

7 Thurber KA, Banks E, Banwell C, et al. Cohort profile: footprints in time, the Australian longitudinal study of Indigenous children. Int $J$ Epidemiol 2015;44:789-800.

8 Comino E, Craig P, Harris E, et al. The Gudaga study: establishing an Aboriginal birth cohort in an urban community. Aust N Z J Public Health 2010;34:S9-17.

9 Sayers SM, Mackerras D, Singh G, et al. An Australian Aboriginal birth cohort: a unique resource for a life course study of an Indigenous population. A study protocol. BMC Int Health Hum Rights 2003;3:1.

10 Ashman AM, Collins CE, Weatherall L, et al. A cohort of Indigenous Australian women and their children through pregnancy and beyond: the Gomeroi gaaynggal study. J Dev Orig Health Dis 2016;7:357-68.

11 Maple-Brown L, Lee I-L, Longmore D, et al. Pregnancy and neonatal diabetes outcomes in remote Australia: the Pandora study-an observational birth cohort. Int J Epidemiol 2019;48:307-18.

12 Najman JM, Bor W, O'Callaghan M, et al. Cohort profile: the MaterUniversity of Queensland study of pregnancy (MUSP). Int J Epidemiol 2005;34:992-7.

13 Straker L, Mountain J, Jacques A, et al. Cohort profile: the Western Australian pregnancy cohort (Raine) Study-Generation 2. Int $J$ Epidemiol 2017;46:dyw308-5.

14 Townsend ML, Riepsamen A, Georgiou C, et al. Longitudinal intergenerational birth cohort designs: a systematic review of Australian and New Zealand studies. PLoS One 2016;11:e0150491.

15 Najman JM, Alati R, Bor W, et al. Cohort profile update: the MaterUniversity of Queensland study of pregnancy (MUSP). Int J Epidemiol 2015;44:78-78f.

16 Callaway LK, Prins JB, Chang AM, et al. The prevalence and impact of overweight and obesity in an Australian obstetric population. Med $J$ Aust 2006;184:56-9.

17 Khan TK, Palmer DJ, Prescott SL. In-Utero exposures and the evolving epidemiology of paediatric allergy. Curr Opin Allergy Clin Immunol 2015:15:402-8.

18 Grieger JA, Clifton VL, Tuck AR, et al. In utero programming of allergic susceptibility. Int Arch Allergy Immunol 2016;169:80-92.

19 Grieger JA, Grzeskowiak LE, Clifton VL. Preconception dietary patterns in human pregnancies are associated with preterm delivery. J Nutr 2014:144:1075-80.

20 Cameron CM, Scuffham PA, Spinks A, et al. Environments for healthy living (EFHL) Griffith birth cohort study: background and methods. Matern Child Health J 2012;16:1896-905.

21 Government,Q. Births by hospital, in open data portal, 2019. Available: https://www.data.qld.gov.au/dataset/births-by-hospital/ resource/d3d0feaa-8929-4cb6-9fd7-eb9f40f21bcb

22 Harris PA, Taylor R, Thielke R, et al. Research electronic data capture (REDCap)-A metadata-driven methodology and workflow process for providing translational research informatics support. J Biomed Inform 2009;42:377-81.

23 Harris PA, Taylor R, Minor BL, et al. The REDCap Consortium: building an international community of software platform partners. $J$ Biomed Inform 2019;95:103208.

24 Welfare, A.I.o.H.a. Australia's mothers and babies data visualisations, 2020. Available: https://www.aihw.gov.au/reports/mothers-babies/ australias-mothers-babies-data-visualisations/contents/stillbirthsand-neonatal-deaths

25 Nohr EA, Liew Z. How to investigate and adjust for selection bias in cohort studies. Acta Obstet Gynecol Scand 2018;97:407-16.

26 Golding J, Birmingham K. Enrollment and response rates in a longitudinal birth cohort. Paediatr Perinat Epidemiol 2009;23:73-85.

$27 \mathrm{Ng} \mathrm{S}-\mathrm{K}$, Scott R, Scuffham PA. Contactable non-responders show different characteristics compared to lost to follow-up participants: insights from an Australian longitudinal birth cohort study. Matern Child Health J 2016;20:1472-84.

28 Althubaiti A. Information bias in health research: definition, pitfalls, and adjustment methods. J Multidiscip Healthc 2016;9:211-7.

29 The National Health and Medical Research Council. National statement on ethical conduct in human research 2007. Canberra: National Health and Medical Research Council, Commonwealth of Australia, 2018.

30 Ferguson KT, Cassells RC, MacAllister JW, et al. The physical environment and child development: an international review. Int $J$ Psychol 2013;48:437-68.

31 Wang E, Glazer KB, Howell EA, et al. Social determinants of pregnancy-related mortality and morbidity in the United States: a systematic review. Obstet Gynecol 2020;135:896-915. 
32 McMichael AJ, Vimpani GV, Robertson EF, et al. The Port Pirie cohort study: maternal blood lead and pregnancy outcome. J Epidemiol Community Health 1986;40:18-25.

33 Magarey AM, Daniels LA, Boulton TJ, et al. Predicting obesity in early adulthood from childhood and parental obesity. Int $J$ Obes Relat Metab Disord 2003;27:505-13.

34 Carmichael A, Williams HE, Picot SG. Growth patterns, health and illness in preschool children from a multi-ethnic, poor socioeconomic status municipality of Melbourne. J Paediatr Child Health 1990;26:136-41.

35 Sayers SM, Mackerras D, Singh GR. Cohort profile: the Australian aboriginal birth cohort (ABC) study. Int J Epidemiol 2017;46:dyw291-1383.

36 Douglas RM, Woodward A, Miles $\mathrm{H}$, et al. A prospective study of proneness to acute respiratory illness in the first two years of life. Int J Epidemiol 1994;23:818-26.

37 Giles LC, Whitrow MJ, Rumbold AR, et al. Growth in early life and the development of obesity by age 9 years: are there critical periods and a role for an early life stressor? Int J Obes 2013;37:513-9.

38 Trevillian LF, Ponsonby A-L, Dwyer T, et al. Infant sleeping environment and asthma at 7 years: a prospective cohort study. Am J Public Health 2005;95:2238-45.

39 Loke YJ, Novakovic B, Ollikainen M, et al. The peri/postnatal epigenetic twins study (pets). Twin Res Hum Genet 2013;16:13-20.

40 Hutchinson D, Wilson J, Allsop S, et al. Cohort profile: the triple B pregnancy cohort study: a longitudinal study of the relationship between alcohol, tobacco and other substance use during pregnancy and the health and well-being of Australian children and families. Int J Epidemiol 2018;47:26-7.

41 Hure AJ, Collins CE, Giles WB, et al. Protocol for the women and their children's health (Watch) study: a cohort of pregnancy and beyond. J Epidemiol 2012;22:267-75.

42 Ashman AM, Collins CE, Weatherall L, et al. A cohort of Indigenous Australian women and their children through pregnancy and beyond: the Gomeroi gaaynggal study. J Dev Orig Health Dis 2016;7:357-68.

43 de Silva-Sanigorski AM, Waters E, Calache H, et al. Splash!: a prospective birth cohort study of the impact of environmental, social and family-level influences on child oral health and obesity related risk factors and outcomes. BMC Public Health 2011;11:505.

44 Johnson S, Carpenter L, Amezdroz E, et al. Cohort profile: the VicGeneration (VicGen) study: an Australian oral health birth cohort. Int J Epidemiol 2017;46:29-30.

45 Hagemann E. The ORIGINS Project. In: Pre-emptive medicine: public health aspects of developmental origins of health and disease. Springer, 2019: 99-116.

46 Juniper EF, O'Byrne PM, Guyatt GH, et al. Development and validation of a questionnaire to measure asthma control. Eur Respir $J$ 1999;14:902-7.

47 Cella D, Choi SW, Condon DM, et al. PROMIS ${ }^{\circledR}$ adult health profiles: efficient short-form measures of seven health domains. Value Health 2019;22:537-44.

48 Monk TH, Buysse DJ, Kennedy KS, et al. Measuring sleep habits without using a diary: the sleep timing questionnaire. Sleep 2003;26:208-12.

49 Morin CM, Belleville G, Bélanger L, et al. The insomnia severity index: psychometric indicators to detect insomnia cases and evaluate treatment response. Sleep 2011;34:601-8.

50 Roenneberg T, Wirz-Justice A, Merrow M. Life between clocks: daily temporal patterns of human chronotypes. J Biol Rhythms 2003;18:80-90.
51 Adhikari P, Cooper-Stanbury M. Australian National infant feeding survey, 2018. Available: http://dx.doi.org/10.26193/LD6OFT.

52 Brown WJ, Burton NW, Marshall AL, et al. Reliability and validity of a modified self-administered version of the active Australia physical activity survey in a sample of mid-age women. Aust N Z J Public Health 2008;32:535-41.

53 Craig CL, Marshall AL, Sjöström M. International physical activity questionnaire: 12 -country reliability and validity. Med Sci Sports Eerc 2003;35:1381-95.

54 Rosenberg DE, Bull FC, Marshall AL, et al. Assessment of sedentary behavior with the International physical activity questionnaire. J Phys Act Health 2008;5:S30-44.

55 Statisitics ABo. National health survey questionnaire, 2018.

56 Wendy JB, Toby P. Physical activity in mid-age and older women: lessons from the Australian longitudinal study on women's health. Kinesiology Review 2016;5:87-97.

$57 \mathrm{He} \mathrm{C}$, Salonen H, Ling X, et al. The impact of flood and post-flood cleaning on airborne microbiological and particle contamination in residential houses. Environ Int 2014;69:9-17.

58 Hopkins C, Gillett S, Slack R, et al. Psychometric validity of the 22item sinonasal outcome test. Clin Otolaryngol 2009;34:447-54.

59 Richardson JRJ, Peacock SJ, Hawthorne G, et al. Construction of the descriptive system for the assessment of quality of life AQoL-6D utility instrument. Health Qual Life Outcomes 2012;10:38.

60 O'Donnell LJ, Virjee J, Heaton KW. Detection of pseudodiarrhoea by simple clinical assessment of intestinal transit rate. BMJ 1990;300:439-40.

61 Agachan F, Chen T, Pfeifer J, et al. A constipation scoring system to simplify evaluation and management of constipated patients. Dis Colon Rectum 1996;39:681-5.

62 Funk JL, Rogge RD. Testing the ruler with item response theory: increasing precision of measurement for relationship satisfaction with the couples satisfaction index. J Fam Psychol 2007;21:572-83.

63 Lovibond PF, Lovibond SH. The structure of negative emotional states: comparison of the depression anxiety stress scales (DASS) with the Beck depression and anxiety inventories. Behav Res Ther 1995;33:335-43.

64 Clarke DE, Kuhl EA. Dsm-5 cross-cutting symptom measures: a step towards the future of psychiatric care? World Psychiatry 2014;13:314-6.

65 Herdman M, Gudex C, Lloyd A, et al. Development and preliminary testing of the new five-level version of EQ-5D (EQ-5D-5L). Qual Life Res 2011;20:1727-36.

66 Collins CE, Boggess MM, Watson JF, et al. Reproducibility and comparative validity of a food frequency questionnaire for Australian adults. Clin Nutr 2014;33:906-14.

67 Baessler K, O'Neill SM, Maher CF, et al. Australian pelvic floor questionnaire: a validated interviewer-administered pelvic floor questionnaire for routine clinic and research. Int Urogynecol J Pelvic Floor Dysfunct 2009;20:149-58.

68 Bennett PJ, Patterson C, Wearing S, et al. Development and validation of a questionnaire designed to measure foot-health status. J Am Podiatr Med Assoc 1998;88:419-28.

69 Bruwer B, Emsley R, Kidd M, et al. Psychometric properties of the multidimensional scale of perceived social support in youth. Compr Psychiatry 2008;49:195-201.

70 Holmes TH, Rahe RH. The social Readjustment rating scale. $J$ Psychosom Res 1967;11:213-8.

71 Cox JL, Holden JM, Sagovsky R. Detection of postnatal depression. development of the 10-item Edinburgh postnatal depression scale. Br J Psychiatry 1987;150:782-6. 